\title{
Bionanofabrication of Metallic and Semiconductor Nanoparticle Arrays Using S-Layer Protein Lattices with Different Lateral Spacings \& Geometries
}

Sonny. S. Mark, ${ }^{* \dagger}$ Magnus Bergkvist,${ }^{\dagger}$ Xin Yang, ${ }^{\S}$ Leonardo M. Teixeira, ${ }^{\dagger}$ Parijat Bhatnagar, ${ }^{\dagger}$ Esther R. Angert, ${ }^{\dagger}$ and Carl. A. Batt ${ }^{\$}$

${ }^{\dagger}$ Department of Microbiology, ${ }^{\ddagger}$ Department of Biomedical Engineering, and ${ }^{\S}$ Department of Food Science at Cornell University, Ithaca, New York 14853, "Present Address: Nanobiotechnology Center at Cornell University, Ithaca, New York 14853.

Supporting Information 
(a)

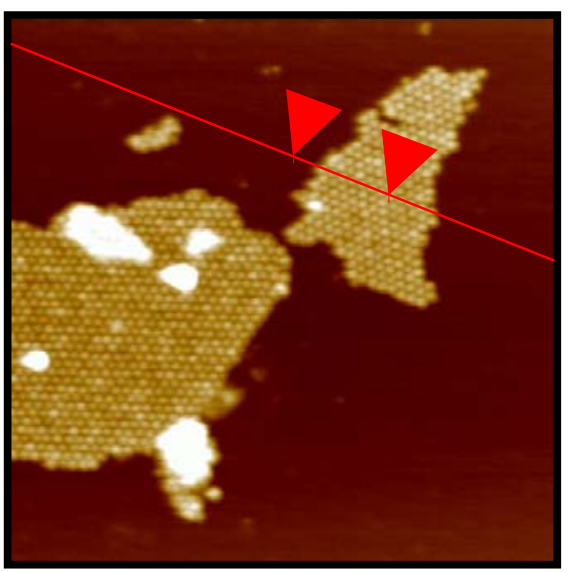

(c)

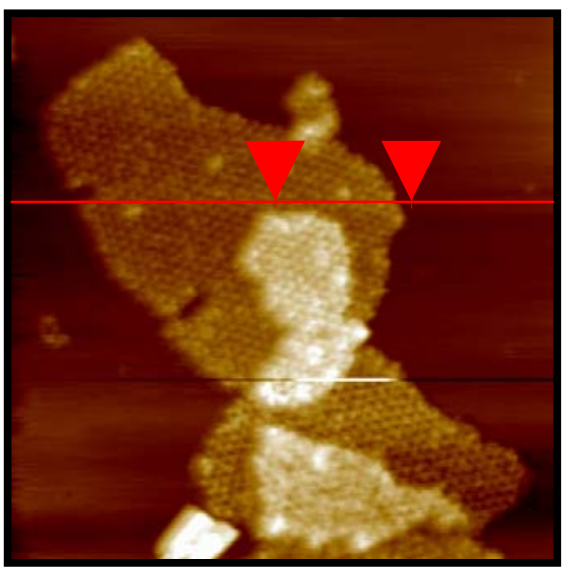

(b)

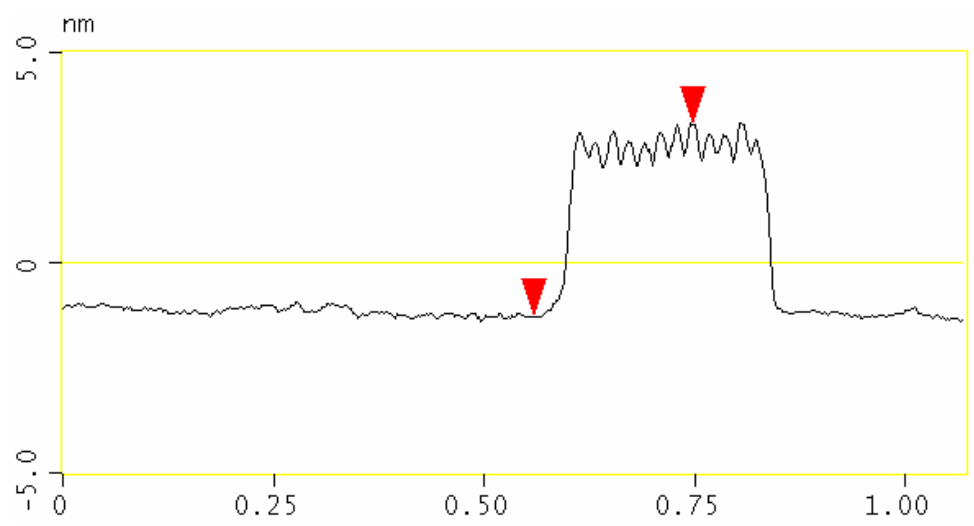

(d)

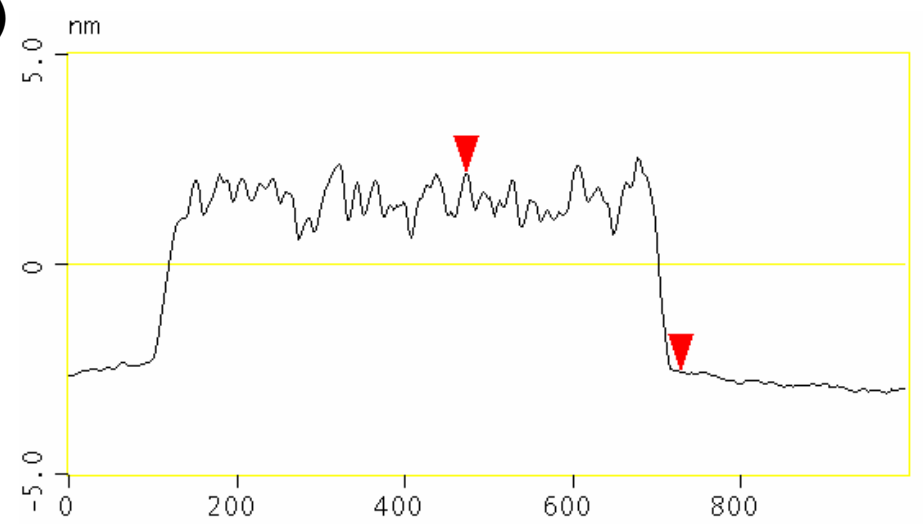

Figure S1. Determination of monolayer thickness of HPI and SAS S-layers by atomic force microscopy (AFM). (a) AFM height image of HPI S-layer $(1 \mu \mathrm{m} \times 1 \mu \mathrm{m}$ scan area). (b) Section analysis profile across the area marked by the red line in the height image in (a). The vertical distance between the red arrowheads is $4.6 \mathrm{~nm}$. (c) AFM height image of SAS S-layer $(1 \mu \mathrm{m} \times 1 \mu \mathrm{m}$ scan area). (d) Section analysis profile across the area marked by the red line in the height image in (c). The vertical distance between the red arrowheads is $4.8 \mathrm{~nm}$. 
<smiles>O=C(O)CC(O)(CC(=O)O)C(=O)O</smiles>

Citric Acid<smiles>O=C(O)CCS</smiles>

3-Mercaptopropionic acid<smiles>CN(C)CCS</smiles>

2-(Dimethylamino)ethanethiol<smiles>O=C(O)CCCCCCCS</smiles>

7-Carboxy-1-heptanethiol<smiles>O=C(O)CCCCCCCCCCCCCCCS</smiles>

16-Mercaptohexadecanoic acid

Chart S1. Chemical structures of the nanoparticle capping ligands used in this study. 


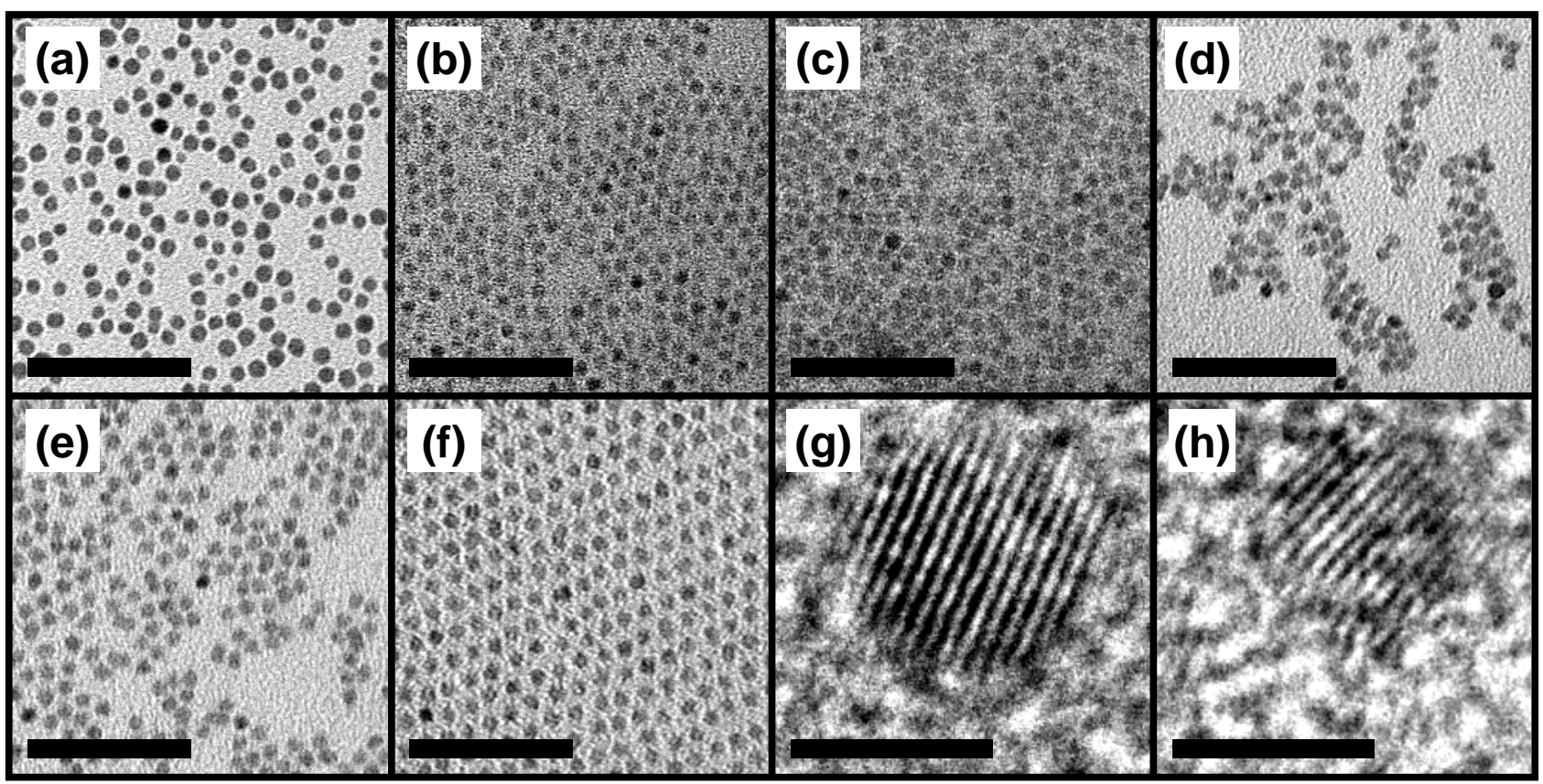

(i)

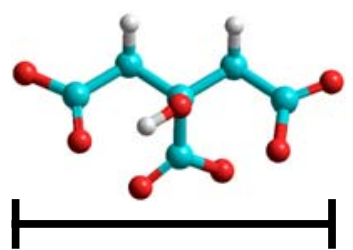

CA: $7.3 \AA$

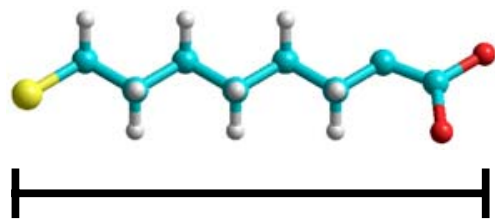

CHT: $11.6 \AA$

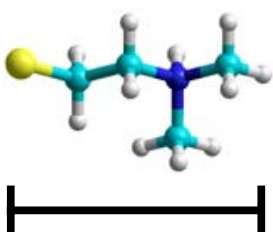

DMAET: $6.1 \AA$

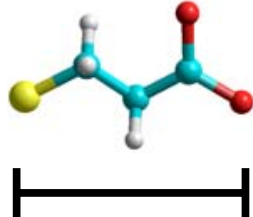

MPA: $5.3 \AA$<smiles>C=C(C)C(C)=C(C)C(C)=C(C)C(C)=C(C)C(C)=C(C)C(C)=C(C)C(C)=C(C)C(C)=C(C)C(=O)O</smiles>

MHA: $21.6 \AA$

Figure S2. Electron micrographs of gold nanoparticles (NPs) and unmodified/modified CdSe/ZnS quantum dots (QDs). Brightfield TEM images of clusters of (a) citrate-capped Au NPs, (b) unmodified (TOP/TOPO-capped) QDs, (c) DMAET-QDs, (d) C3-QDs, (e) C8 QDs, and (f) C16 QDs. HRTEM images of individual (g) unmodified and (h) C16-modified QDs showing well-resolved crystalline lattice planes. (i) Calculated end-to-end dimensions of molecular models of the fully extended form of the capping ligands employed in this study. The ball and stick models depict the ligands in their ionized states and are drawn to scale with standard bond angles/lengths. Coloring scheme: $\mathrm{C}$, cyan; $\mathrm{H}$, white; $\mathrm{N}$, blue; $\mathrm{O}$, red; S, yellow. CA, Citric acid; DMAET, 2(Dimethylamino)ethanethiol; MPA, 3-Mercaptopropionic acid; CHT, 7-Carboxy-1-heptanethiol; MHA, 16-Mercaptohexadecanoic acid. (a)-(f): Scale bar $=50 \mathrm{~nm}$. (g) and (h): Scale bar $=5 \mathrm{~nm}$. 


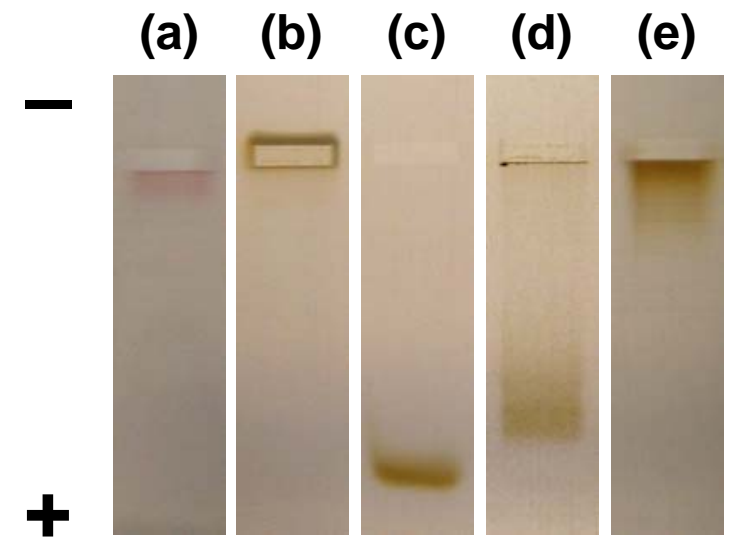

Figure S3. Agarose gel electrophoresis analysis of gold nanoparticles (NPs) and water-soluble quantum dots (QDs). (a) Citrate-stabilized Au NPs. (b) DMAET-QDs. (c) C3-QDs. (d) C8-QDs. (e) C16-QDs. All images are taken from the same gel slab and are aligned with respect to the position of the sample well. 
(a)

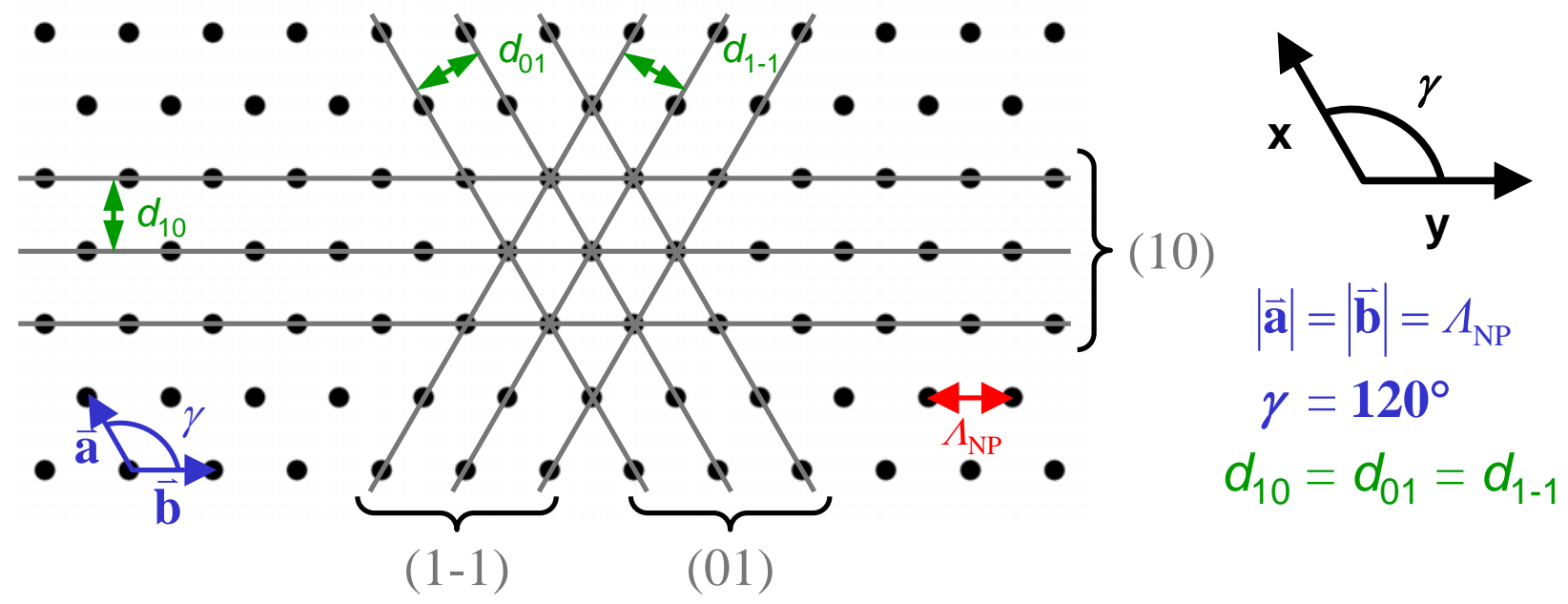

(b)
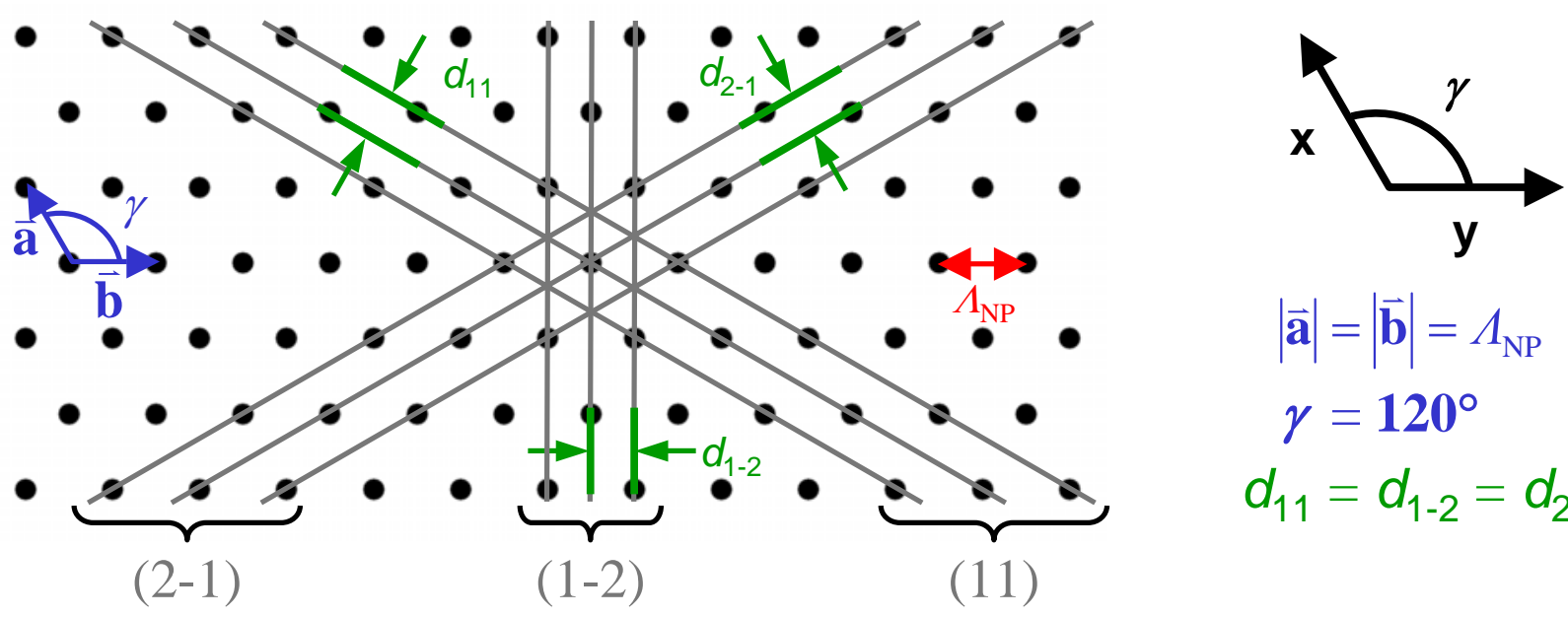

$$
\begin{aligned}
|\overrightarrow{\mathbf{a}}| & =|\overrightarrow{\mathbf{b}}|=\Lambda_{\mathrm{NP}} \\
\gamma & =\mathbf{1 2 0 ^ { \circ }} \\
d_{11} & =d_{1-2}=d_{2-1}
\end{aligned}
$$

(c)

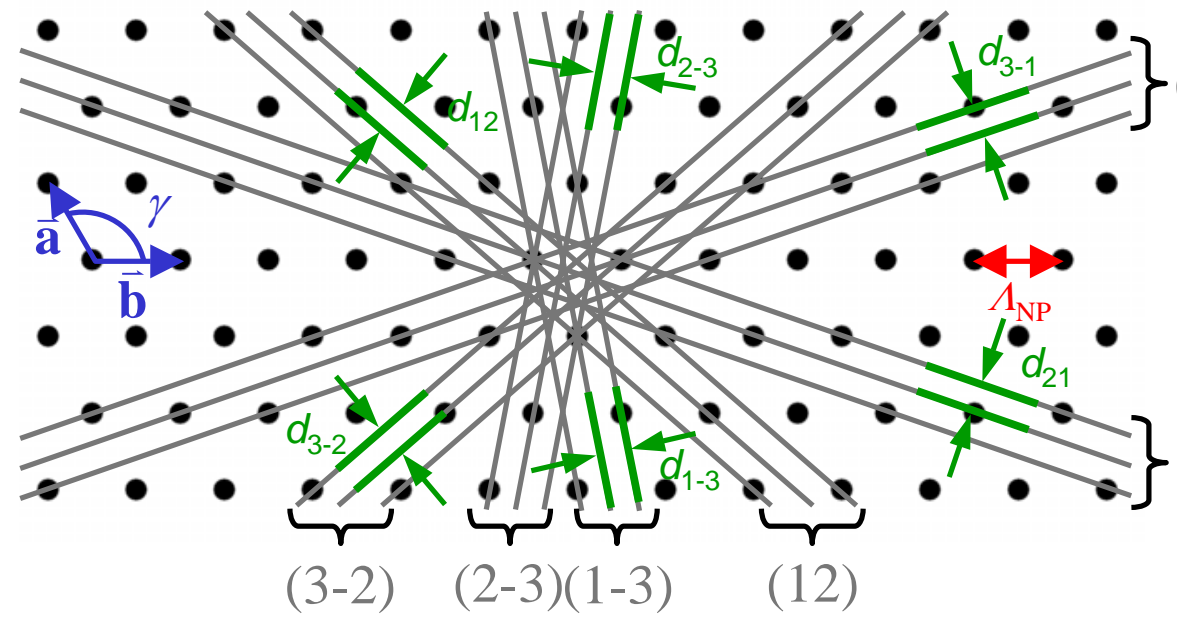

$(3-1)$

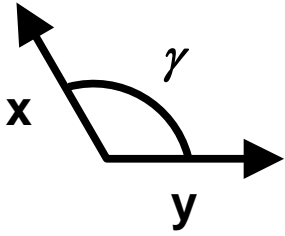

$$
\begin{aligned}
|\overrightarrow{\mathbf{a}}| & =|\overrightarrow{\mathbf{b}}|=\Lambda_{\mathrm{NP}} \\
\gamma & =\mathbf{1 2 0}^{\circ} \\
d_{12} & =d_{1-3}=d_{21} \\
=d_{2-3} & =d_{3-1}=d_{3-2}
\end{aligned}
$$

Figure S4. Schematic illustration of the major lattice orientations in an ideal two-dimensional hexagonal array structure. $\overrightarrow{\mathbf{a}}$ and $\overrightarrow{\mathbf{b}}$ are the primitive hexagonal unit cell vectors. ( $h k$ ) is the two-index specification of a set of parallel lines in the 2-D array, analogous to the Miller indices $(h k l)$ of a set of planes in a 3-D crystal. $d_{\mathrm{hk}}$ ("lattice spacing") is the orthogonal distance between two lattice lines of a given set. $\Lambda_{\mathrm{NP}}$ ("lattice constant") is the interparticle nearest-neighbor (center-to-center) distance. (a) The set of lattice lines with $d_{\mathrm{hk}}$ $=\Lambda_{\mathrm{NP}}\left(\cos \left(30^{\circ}\right)\right)$. (b) The set of lattice lines with $d_{\mathrm{hk}}=\Lambda_{\mathrm{NP}} / 2$. (c) The set of lattice lines with $d_{\mathrm{hk}}=\Lambda_{\mathrm{NP}}\left(\cos \left(30^{\circ}\right) / \sqrt{ } 7\right)$. 

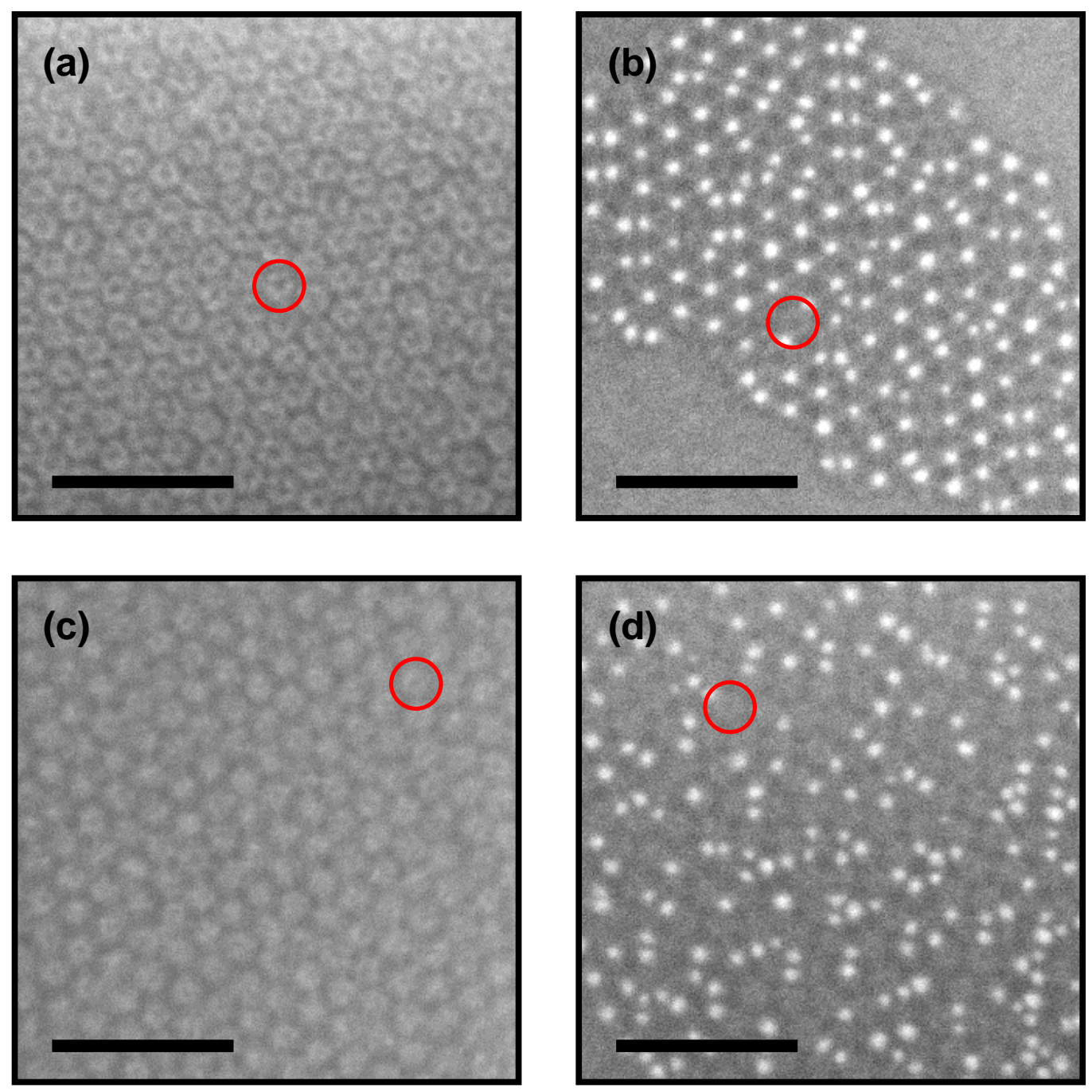

Figure S5. Scanning electron microscopy (SEM) analysis of gold nanoparticle (Au NP) adsorption on extracellular versus intracellular face of HPI S-layer. (a) Native HPI S-layer extracellular face. The red circle outlines a representative hexameric protein core unit displaying the large-diameter central pore characteristic of the extracellular face of the S-layer. (b) Au NPs bound to HPI S-layer extracellular face (Type H1 adsorption pattern). The red circle outlines a representative hexameric protein core unit displaying the largediameter central pore characteristic of the extracellular face of the S-layer. (c) Native HPI S-layer intracellular face. The red circle outlines a representative hexameric protein core unit displaying the smalldiameter central pore characteristic of the intracellular face of the S-layer. (d) Au NPs bound to HPI S-layer intracellular face (Type $\mathrm{H} 2$ adsorption pattern). The red circle outlines a representative hexameric protein core unit displaying the small-diameter central pore characteristic of the intracellular face of the S-layer. For all images, scale bar $=100 \mathrm{~nm}$. 


\section{Case 1}

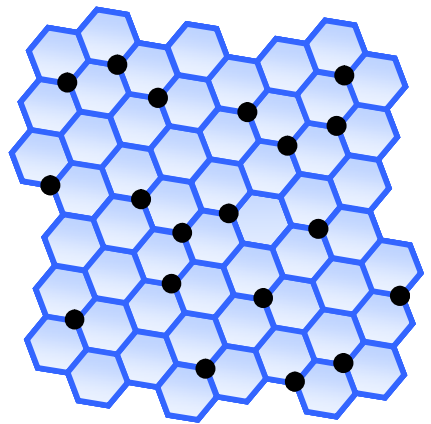

(1)

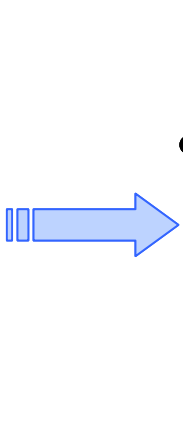

(2)

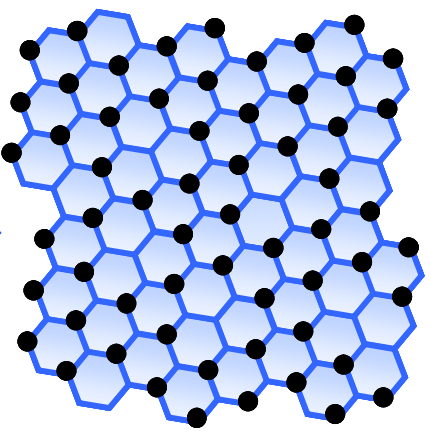

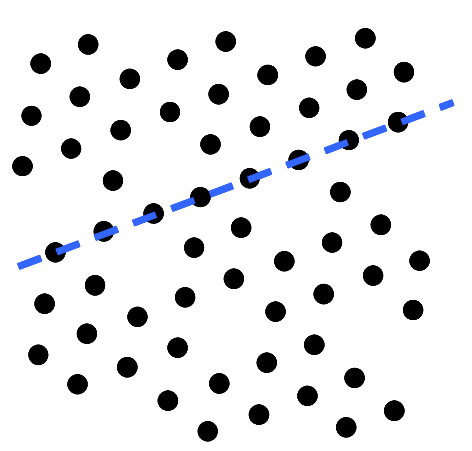

\section{Observed Adsorption Pattern ( No Defective Lattice Lines Present)}

\section{Case 2}

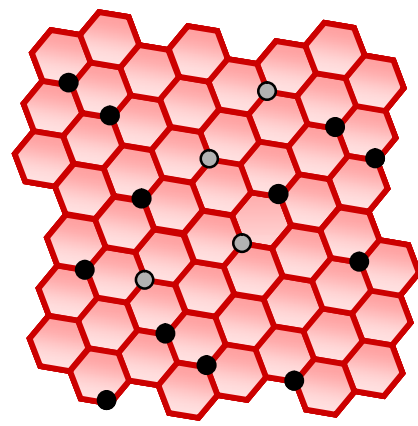

(1)

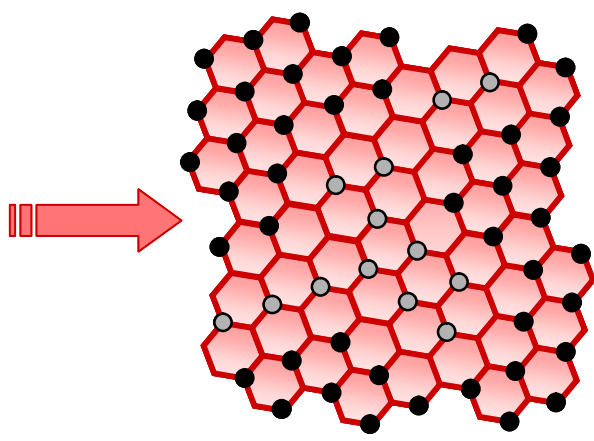

(2)

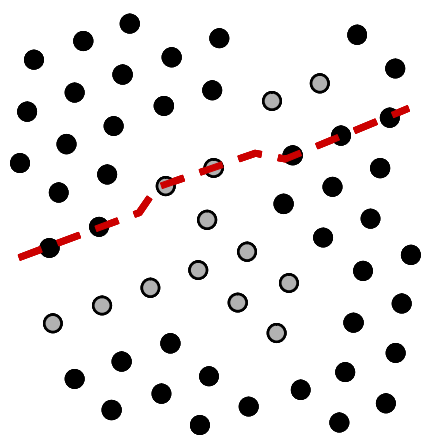

Observed Adsorption Pattern (Defective Lattice Lines Are Present)

Figure S6. Schematic illustration of the hypothetical progression of nanoparticle binding to the vertex regions of the HPI S-layer. Different adsorption patterns arise, depending on the initial adsorption of particles relative to each other. Gray dots represent particles adsorbed at positions located an odd number of vertex regions away from any adjacent particles represented by black dots. (1) and (2) represent earlier and later phases of nanoparticle adsorption, respectively.

Case 1: All nanoparticles adsorb an even number of vertex regions away from other nanoparticles on the S-layer protein sheet. In this case, no apparently "misaligned" particles are visible when lattice lines are drawn over the resulting adsorption pattern (cf. dashed blue line).

Case 2: Nanoparticles represented by gray dots adsorb onto the S-layer protein in such a way that other nanoparticles (black dots) are located an odd number of vertex regions away from them. Here, the gray particles appear to be "misaligned" relative to the black particles when lattice lines are drawn over the resulting adsorption pattern ( $c f$. dashed red line). 
(a)
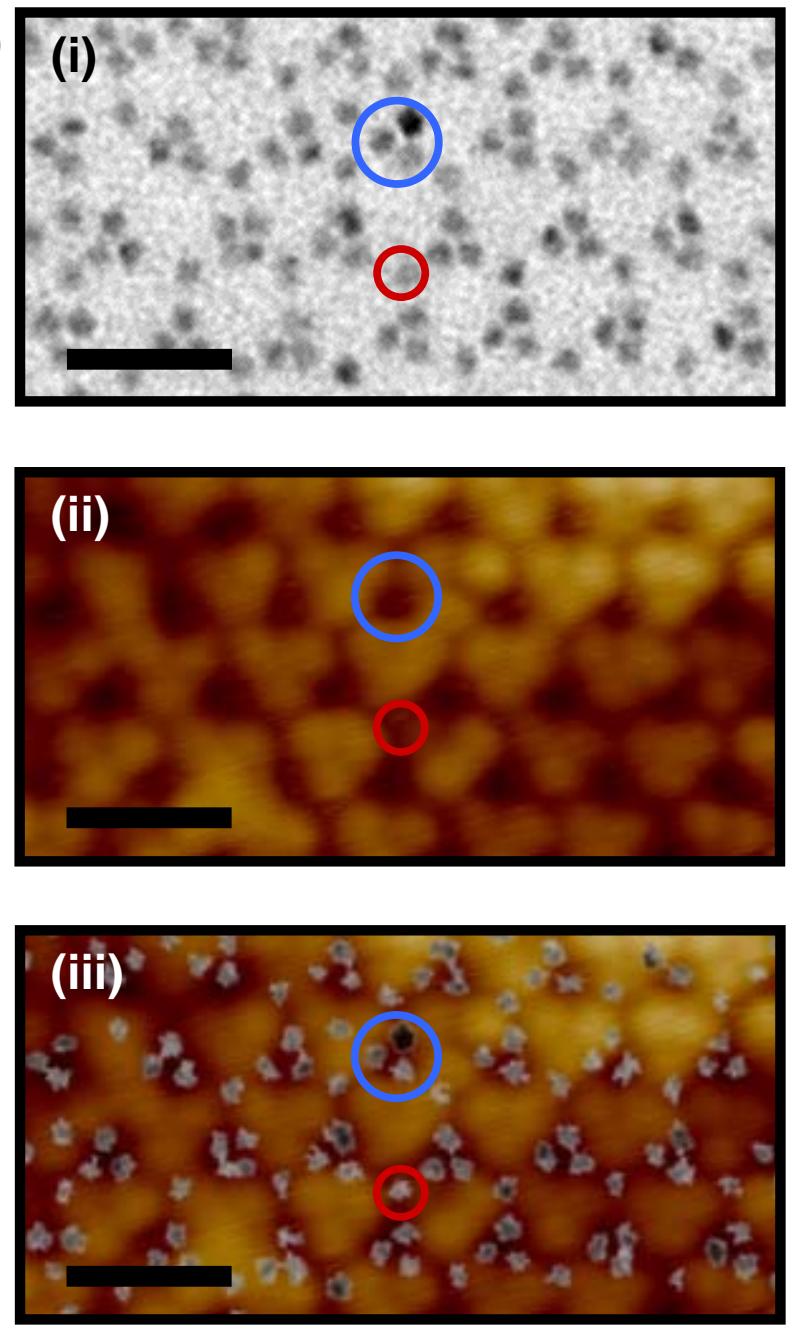

(b)
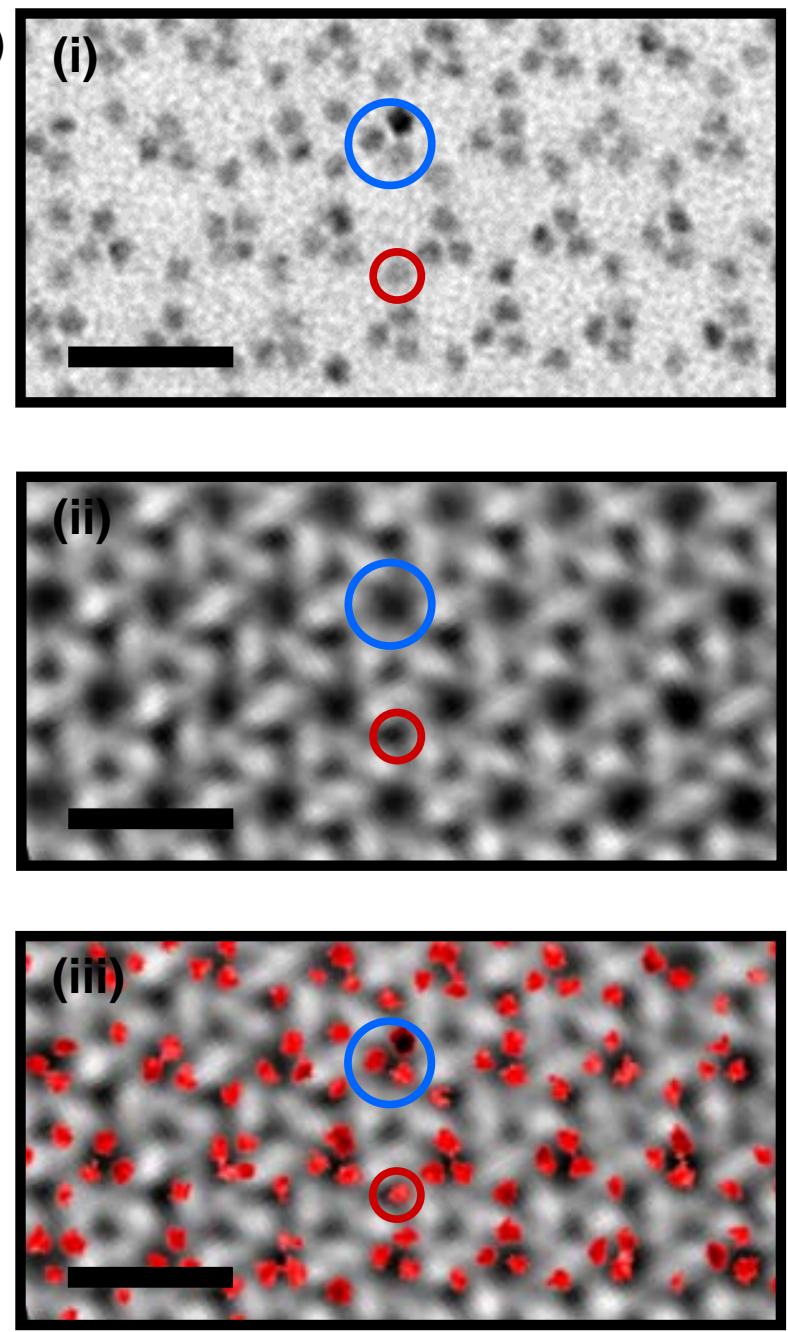

Figure S7. Hypothetical model depicting C8-QDs bound to the inner face of the SAS S-layer. (a) The image shown in (iii) is an overlay of a TEM image of the biotemplated C8-QDs (i) onto an AFM image of the inner face of the native SAS S-layer (ii). (b) The image shown in (iii) is an overlay of a TEM image of the biotemplated C8-QDs (i) onto a noise-filtered (negative-stain) TEM image of the inner face of the native SAS S-layer (ii). Blue and red circles indicate identical points of reference before and after overlaying the separate source images. In the overlay image (iii) shown in (b), the color balance has been adjusted so that the C8-QDs appear mostly red. Scale bar $=25 \mathrm{~nm}$ in all images. To produce the overly images, the source images of the biotemplated C8-QDs were digitally thresholded to remove the speckled carbon-coated formvar TEM grid background. 
(a)

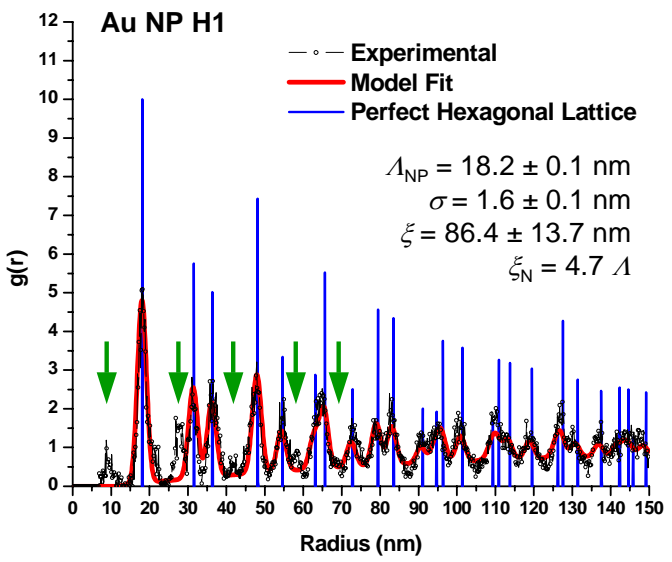

(c)

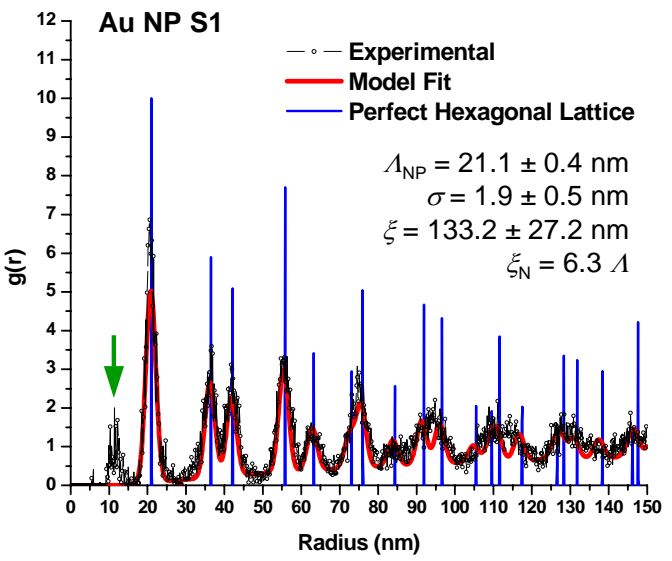

(e)

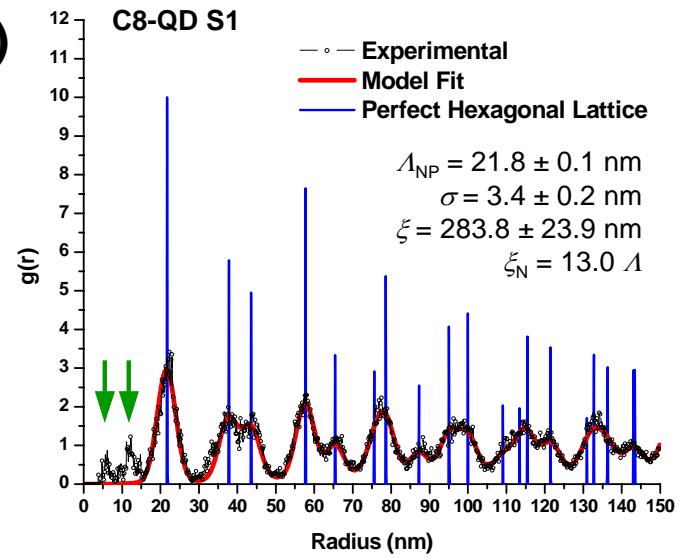

(b)

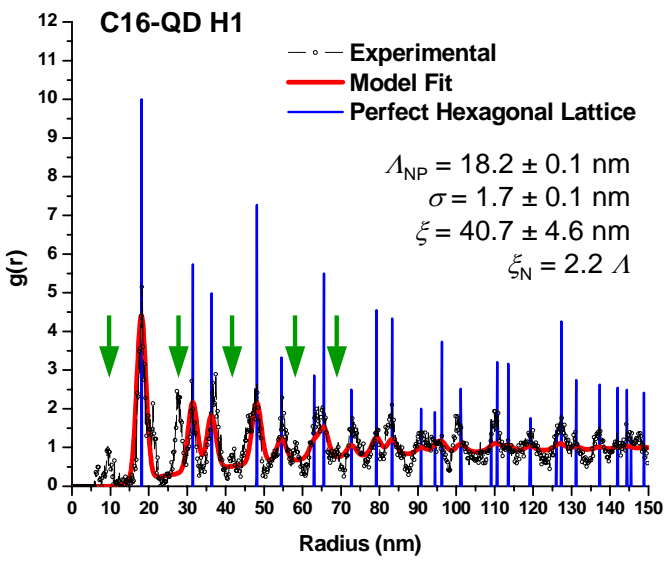

(d)

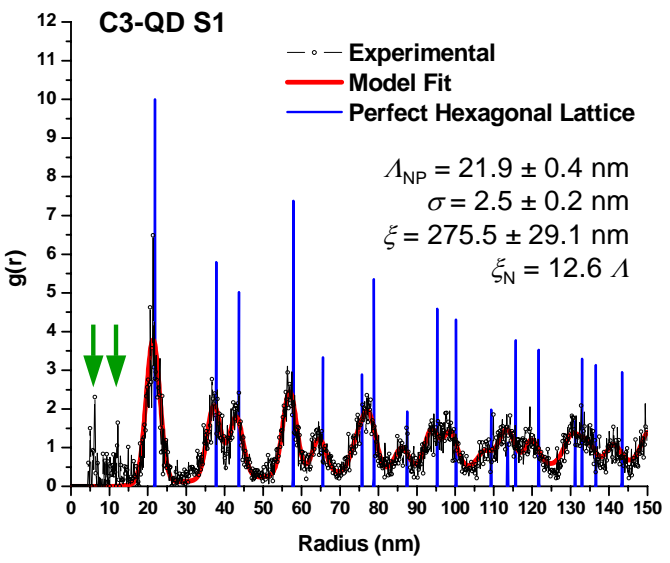

(f)

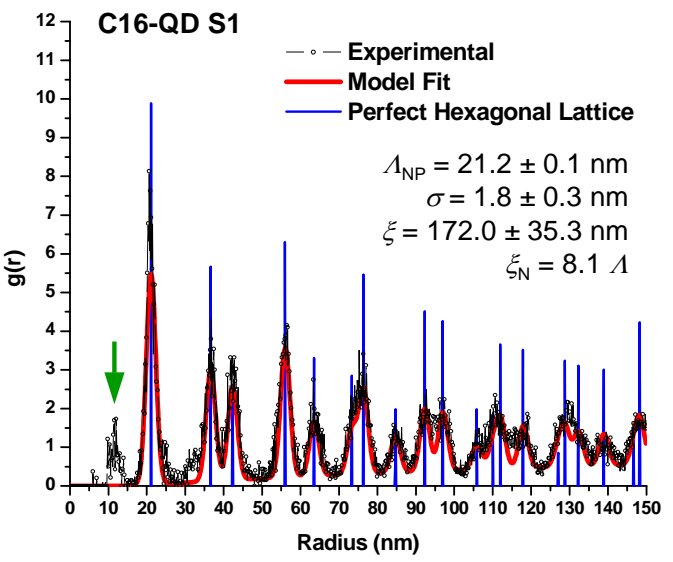

Figure S8. Two-dimensional pair correlation functions, $g(r)$, for six different Au nanoparticle (NP) and CdSe/ZnS quantum dot (QD) hexagonal array patterns biotemplated on the HPI and SAS S-layers. The representative PCF plots correspond to the following nanoparticle adsorption patterns: (a) Au NP H1. (b) C16-QD H1. (c) Au NP-QD S1. (d) C3-QD S1. (e) C8-QD S1. (f) C16-QD S1. Dashed black lines $\left(g(r)_{\exp }\right)$ with circles represent experimental $g(r)$ values. Solid red lines $\left(g(r)_{\text {fit }}\right)$ are a three-parameter fit of the experimental data to the model in Eq. (2) ( $\Lambda_{\mathrm{NP}}=$ lattice spacing, $\sigma=$ Gaussian peak half-width, $\xi=$ translational correlation length). Solid blue lines $\left(g(r)_{\text {hex }}\right)$ are a series of delta function peaks representing the ideal PCF plot for a perfect 2-D hexagonally-packed array of particles with nearest-neighbor (center-to-center) spacing $\Lambda_{\mathrm{NP}} . \xi_{\mathrm{N}}=\xi / \Lambda_{\mathrm{NP}}$ (normalized translational correlation length). Reported values of $\Lambda_{\mathrm{NP}}, \sigma$, and $\xi$ are mean values ( \pm standard deviation) obtained from a set of 3 images taken from different regions of the same sample. Green arrows point to subordinate peaks which cannot be fit to Eq. (2). 